\title{
Pattern of failure after high-dose thoracic radiation for non-small cell lung cancer: the University of Michigan experience
}

\author{
Klaudia U. Hunter $\cdot$ Feng-Ming (Spring) Kong • \\ Indrin J. Chetty • Paul Cronin • Daniel Tatro • \\ Charles Marn • James A. Hayman • \\ Randall K. Ten Haken
}

Received: 29 March 2012 / Accepted: 12 April 2012 / Published online: 26 July 2012

(C) Springer-Verlag (outside the USA) 2012

\begin{abstract}
Purpose Our main purpose is to study the pattern of local failure for patients with non-small cell lung cancer treated with conformal therapy.

Methods This study included patients who failed locally and a matched group without failures after 3D conformal radiation per a radiation dose-escalation trial. Radiation doses ranged from 65.1 to 102.9 Gy in 2.1 Gy fractions, originally computed using an equivalent path length algorithm. The recurrent gross target volumes (RGTV) were contoured. The original and recurrent planning target volume (PTV and RPTV) were generated by $1 \mathrm{~cm}$ uniform expansion from GTV. DVHs and generalized equivalent uniform doses $\left(\mathrm{EUD}=\left\{\Sigma_{i}\left(d_{i}\right)^{a}\right\}^{1 / a}\right)$ were computed. Marginal failures were defined for RGTVs covered by the original 10 to $90 \%$ isodose surfaces.

Results There were no significant differences between the failed and control groups with regard to average original GTV volumes, GTV and PTV doses, and minimum PTV doses. Of the 18 RGTVs, four had marginal failure, 12 failed mostly within, and two failed outside of the original PTV. The mean EUDs were 57.1 Gy (95\% confidence
\end{abstract}

K. U. Hunter $\cdot$ F.-M. Kong $(\bowtie) \cdot$ I. J. Chetty $\cdot$ D. Tatro $•$

J. A. Hayman • R. K. Ten Haken

Department of Radiation Oncology, University of Michigan,

Box 0010, UH-B2C490 1500 E. Medical Center Dr.,

Ann Arbor, MI 48109, USA

e-mail: Fengkong@med.umich.edu

P. Cronin · C. Marn

Department of Radiology, University of Michigan,

Ann Arbor, MI, USA

F.-M. Kong · C. Marn

Veteran Affair Hospital,

Ann Arbor, MI, USA interval (CI) 43.9-70.6) and 47.5 Gy (95\% CI 33.7-61.2), for the RGTVs and RPTVs, respectively, significantly below the prescribed doses $(p=0.03)$. EUDs were less than 60 Gy for $39 \%$ of the RGTVs and $56 \%$ of the RPTVs. Conclusions Recurrent tumors had significantly lower doses than the prescribed dose suggesting that some of these failures could have been avoided with modern technology such as 4D $\mathrm{CT}$ simulation and image-guided radiation therapy.

Keywords Non-small cell lung cancer · 3D conformal radiation $\cdot$ Pattern of local failure

\section{Introduction}

Local failure in non-small cell lung cancer continues to be a major problem in the management of these patients. Several retrospective data sets have shown an association between higher treatment dose with improved local control and overall survival [1-3]. We performed a phase I dose-escalation study at the University of Michigan with doses ranging from 65.1 to 102.9 Gy [4-8]. Analysis of this data showed a statistically significant relationship between dose and local control and overall survival. Five-year local control rates were $12 \%$ for patients receiving 63-69 Gy, $35 \%$ for patients receiving 74 $84 \mathrm{~Gy}$, and $49 \%$ for patients receiving $92-103 \mathrm{~Gy}$. The doseresponse relationship was steeper for longer follow-up with the strongest relationship between local control and radiation dose being evident for 5 years of follow-up. However, despite the aggressiveness of this approach, nearly half of patients in the highest dose group still recurred locally. Other investigators have also found high rates of local recurrence despite dose escalation [9-12]. 
The recent presentation of the interim analysis of RTOG 0617 draws new attention to the role of dose escalation in non-small cell lung cancer. During the first interim analysis (median follow-up of 10-11 months), the higher dose arm, $74 \mathrm{~Gy}$, did not improve overall survival compared to the standard dose arm, receiving $60 \mathrm{~Gy}$. Because of the renewed interest in this topic, we have further examined the pattern of failure for patients treated at the University of Michigan on our dose-escalation trial. In this study, we compare the recurrent tumor to the initial target and radiation plan to better understand the factors that contributed to local failure in a prospective trial that used similar radiation technology as RTOG-0617. We hypothesize that local recurrence was secondary to under-dosing areas at risk secondary to the now, out-dated radiation techniques employed during these trials.

\section{Materials and methods}

\section{Study population}

Full details of protocol eligibility have been previously described [4-8]. Briefly, patients with histologically confirmed, new or recurrent stages I-III non-small cell lung cancer (NSCLC) were eligible for this analysis. Patients with prior thoracic radiation, biopsy-proven supraclavicular involvement, documented malignant pleural or pericardial effusions, or noncontiguous involvement of parietal pleura were excluded. There were no minimum requirements for pulmonary function for participation, but patients were required to have an ECOG performance status of $0-2$.

For this analysis, study patients who failed in or near previously irradiated regions and had retrievable CT images from both simulation and follow-up (at the time of first local regional progression per radiology report) were included. A dose-matched group with no evidence of local progression and a minimum of 1 year of follow-up was used for comparison. All patients provided informed consent, and this study was performed in accordance with the Institutional Review Board of the University of Michigan and participating affiliate hospitals.

\section{Chemotherapy}

All patients were treated with radiation alone between 1992 and 1997. From 1997 to 2000, patients with stage III or locally recurrent disease without significant weight loss (no more than $20 \%$ unexplained weight loss in the preceding 6 months) were treated with neoadjuvant chemotherapy. This consisted of two cycles of cisplatin and vinorelbine followed by radiation therapy on day 50 . Upon completing chemotherapy, patients who were not eligible for dose escalation because of an inability to meet normal tissue dose constraints were removed from the study.

Radiation technique

Details of the dose-escalation schema and radiation technique have been published previously [4-8]. Briefly, patients were simulated in the supine position, freebreathing with a foam cradle for immobilization. CT scans with intravenous contrast were performed for treatment planning with fluoroscopy used to assess internal target motion. Patients treated with neoadjuvant chemotherapy underwent a treatment planning simulation both before and after receiving chemotherapy.

The gross tumor volume (GTV) was defined as the primary tumor and any involved nodal disease (as documented by biopsy or enlargement $>1 \mathrm{~cm}$ on imaging). The GTV was uniformly expanded by $0.5 \mathrm{~cm}$ to create the clinical target volume (CTV). An additional $0.5-1.0 \mathrm{~cm}$ was added to account for internal target motion secondary to respiration to create the internal target volume (ITV). A final expansion of $0.5 \mathrm{~cm}$ was added for setup error to create the planning target volume (PTV). For patients who received chemotherapy, the post-chemotherapy volume was used to define the primary tumor GTV, and all initially involved nodal disease was targeted regardless of response to chemotherapy. For patients with no residual disease after chemotherapy, the epicenter of the initial area of disease was identified and expanded by $1.5 \mathrm{~cm}$ to create a CTV. Elective nodal irradiation was not performed.

All patients were treated once daily. The dose was prescribed to a point at the isocenter (100\% isodose line) with at least the $95 \%$ isodose line covering the entire PTV. Plans consisted of two to seven beams treated with 6-25 MV photons. Patients were treated with doses ranging from 65.1 to 102.9 Gy in 2.1 Gy per fraction. The details of the dose-escalation schema were previously published [4-8]. Equivalent path length dose calculation was used for tissue heterogeneity correction.

Follow-up and evaluation of recurrent disease

Patients were seen in follow-up 1 month after the completion of treatment and every 3 months for the first 2 years. They were subsequently seen every 4 months during the third year, every 6 months during the fourth and fifth years, and annually thereafter. Patients underwent a chest X-ray at each visit and a thoracic CT every 6 months. For patients with disease detectable on bronchoscopy, a 6-month followup bronchoscopy was performed.

For patients with recurrent disease, follow-up CT imaging was registered with the original treatment planning CT scan using a mutual information algorithm. The recurrent 
Table 1 Dosimetric comparison between patients grouped by recurrence status

\begin{tabular}{|c|c|c|c|c|c|c|}
\hline & $\begin{array}{l}\text { Prescription } \\
\text { dose (Gy) }\end{array}$ & GTV volume (cc) & $\begin{array}{l}\text { GTV min } \\
\text { dose (Gy) }\end{array}$ & GTV EUD (Gy) & $\begin{array}{l}\text { PTV min } \\
\text { dose (Gy) }\end{array}$ & PTV EUD (Gy) \\
\hline \multicolumn{7}{|l|}{ Group with recurrence } \\
\hline Mean & 74.3 & 138.0 & 73.0 & 76.2 & 66.4 & 75.8 \\
\hline Median & 69.3 & 119 & 70.2 & 71.7 & 65.3 & 71.7 \\
\hline Standard deviation & 11.3 & 126.8 & 12.0 & 11.3 & 11.2 & 11.4 \\
\hline $95 \%$ CI & $69.1-79.5$ & $79.4-196.6$ & $67.4-78.5$ & $71.0-81.4$ & $61.2-71.6$ & $70.5-81.1$ \\
\hline \multicolumn{7}{|l|}{ Group without recurrence } \\
\hline Mean & 77.5 & 118.4 & 76.9 & 80.5 & 71.1 & 79.3 \\
\hline Median & 75.6 & 66.3 & 74.4 & 76.6 & 68.3 & 75.7 \\
\hline Standard deviation & 12.8 & 176.0 & 13.9 & 13.7 & 15.9 & 15.1 \\
\hline $95 \%$ CI & $71.5-83.4$ & $37.1-199.7$ & $70.5-83.3$ & $74.2-86.8$ & $63.7-78.5$ & $72.3-86.3$ \\
\hline $\begin{array}{l}p \text { value comparing mean } \\
\text { value between groups }\end{array}$ & 0.44 & 0.70 & 0.37 & 0.31 & 0.31 & 0.43 \\
\hline
\end{tabular}

GTV (RGTV) was then contoured on the follow-up imaging by an American Board Certified radiation oncologist with 8 years of clinical experience in thoracic oncology (FMK) together with a fellowship-trained thoracic radiologist with 7 years of clinical experience (PC). This volume was expanded by $0.5 \mathrm{~cm}$ to create a recurrent $\mathrm{CTV}$ and another $0.5 \mathrm{~cm}$ to create a recurrent PTV (RPTV). Because the follow-up CT scans were performed during a breath-hold without motion assessment, an accurate ITV could not be generated for the RGTV.

The pattern of failure was characterized by the location of the RGTV relative to the initial radiation treatment plan as evaluated by a radiation oncologist and medical physicist. Recurrences were deemed "within PTV failures" if the majority of the RGTV was covered by the $90 \%$ or higher isodose lines. If the majority of the RGTV was covered by 10 to $90 \%$ isodose lines, this was classified as "marginal failure." Recurrences where the center of RGTV was outside of the original $10 \%$ isodose lines were classified as "outside of PTV failures."

Dose-volume histograms were created using the original treatment plan and the fused data to compare doses to the original and recurrent target volumes. Generalized equivalent uniform doses $\left(\mathrm{EUD}=\left\{\Sigma_{i}\left(d_{i}\right)^{a}\right\}^{1 / a}\right)$ were also computed for each target structure assuming aggressive tumor types $(a=-20)$. Data were analyzed using a two-sided $T$ test with two-sample equal variance.

\section{Results}

Between 1992 and 2002, 122 patients with NSCLC were consented at the University of Michigan Hospital and its affiliates. Excluding patients with major protocol violations (e.g., changes of fraction size), those failing to meet normal tissue dose constraints, and those who received $<63 \mathrm{~Gy}$, a total of 106 patients were evaluable. Eighty-nine patients eventually progressed from NSCLC; progression was noted initially at the local site alone in $37 \%$, at nodal region and local site (1 patient also at distant site) in $6 \%$, at local and distant sites in $11 \%$, and at distant sites only in $46 \%$. Ultimately, $46 \%$ of patients had both local and distant progression, $6 \%$ regional and local distant progressions, $21 \%$ local progression only, and $27 \%$ distant progression only. Therefore, $73 \%$ of patients ultimately had a component of locoregional failure. Of these patients $(n=77), 18$ patients had simulation and follow-up CT scans available for review and dosimetric analysis. These 18 patients along with 18 dose-matched controls without recurrence are presented here. For the 18 patients with locoregional failure, the

Table 2 Dosimetric comparison between the original and recurrent target volumes

\begin{tabular}{|c|c|c|c|c|c|c|c|c|c|c|}
\hline & $\begin{array}{l}\text { GTV } \\
\text { volume } \\
\text { (cc) }\end{array}$ & $\begin{array}{l}\text { GTV } \\
\text { EUD } \\
\text { (Gy) }\end{array}$ & $\begin{array}{l}\text { PTV } \\
\text { EUD } \\
\text { (Gy) }\end{array}$ & $\begin{array}{l}\text { RGTV } \\
\text { volume } \\
\text { (cc) }\end{array}$ & $\begin{array}{l}\text { RGTV } \\
\text { min } \\
\text { dose (Gy) }\end{array}$ & $\begin{array}{l}\text { RGTV } \\
\text { EUD } \\
\text { (Gy) }\end{array}$ & $\begin{array}{l}\% \text { EUD } \\
\text { RGTV/ } \\
\text { PTV }\end{array}$ & $\begin{array}{l}\text { RPTV } \\
\text { min } \\
\text { dose (Gy) }\end{array}$ & $\begin{array}{l}\text { RPTV EUD } \\
\text { (Gy) }\end{array}$ & $\begin{array}{l}\% \text { EUD } \\
\text { RPTV/ } \\
\text { PTV }\end{array}$ \\
\hline Mean & 138.0 & 76.2 & 75.8 & 69.4 & 51.7 & 57.2 & 75 & 39.4 & 47.5 & 63 \\
\hline Median & 119.0 & 71.7 & 71.7 & 36.0 & 64.1 & 67.2 & 97 & 37.2 & 51.8 & 67 \\
\hline $\begin{array}{l}\text { Standard } \\
\text { deviation }\end{array}$ & 126.8 & 11.3 & 11.4 & 113.8 & 28.2 & 28.9 & 35 & 27.1 & 29.8 & 39 \\
\hline $95 \%$ CI & 79.4-196.6 & $71.0-81.4$ & $70.5-81.1$ & $16.8-122.0$ & $38.7-64.7$ & $43.9-70.6$ & $58.5-91.1$ & $26.9-52.0$ & $33.7-61.2$ & $44.8-80.7$ \\
\hline
\end{tabular}


median age was 65 years old, and there were 12 men and six women. For the 18 dose-matched controls, the median age was 68 years old, and there were 13 men and five women. There was a wide distribution of stages in both groups with approximately half of all patients (in either group) with either stage IIIa or IIIb disease.

Eighteen patients had a locoregional recurrence. Twelve out of 18 patients $(67 \%)$ failed within the original PTV with nine $(75 \%)$ failing centrally and three $(25 \%)$ failing peripherally. Four patients $(22 \%)$ experienced a marginal failure. Only two patients (11\%) failed outside of the original PTV. Of note, five of 18 (28\%) failures involved the ipsilateral hilum.

We examined the dosimetric coverage of the original target structures in all study patients. Table 1 shows the GTV volumes and GTV/PTV doses for the original study plans. There was no statistically significant difference between the minimum dose, mean dose, or EUD to the GTV or PTV between the group of patients that had a locoregional recurrence and those that did not.

We also examined the dose given to the volume encompassed by the recurrent disease on the original treatment plan by superimposing the RGTV and RPTV on the original plan. Table 2 shows the doses given to the area of the RGTV and RPTV from the original plan. The mean EUD was 57.1 Gy for RGTV and 47.5 Gy for RPTV (Fig. 1). These are significantly lower than that of original GTV $(p=0.02)$ and PTV $(p=0.002)$. Figure $2 \mathrm{a}, \mathrm{b}$ shows the percent of each recurrent target volume that was covered by the 40-, 60-, and 80-Gy isodose lines. Seven of 18 (39\%) RGTVs and ten of $18(56 \%)$ RPTVs had an EUD less than $60 \mathrm{~Gy}$.

\section{Discussion}

This study investigated the pattern of local failure and demonstrated that $67 \%$ failed mostly within the PTV, $21 \%$ on the margin of the PTV, and $10 \%$ failed outside of the original PTV. The mean EUDs were 57.1 and $47.5 \mathrm{~Gy}$ for the RGTVs and RPTVs respectively, significantly below the prescribed doses $(p=0.03)$. EUDs were less than $60 \mathrm{~Gy}$ for $39 \%$ of the RGTVs and $56 \%$ of the RPTVs.

The cause of locoregional failures in this trial is multifactorial. Half of the patients failed centrally. Considering that majority of these patients received less than $80 \mathrm{~Gy}$ and $>100$ Gy BED is needed to control stage I disease, one may still assume inadequate dose may be the major reason of these failure [13]. However, comparison of these patients with a dose-matched cohort without failure suggests that underdosing of the original GTV/PTV may not be the single cause of these failures. The patients that failed could have been inherently radioresistant, and despite our attempts at dose escalation, these doses may not have been enough. Furthermore, this trial utilized equivalent path length method for dose calculations. This technique could have led to an overestimate of the peripheral tumor dose delivered, and utilizing
Fig. 1 Equivalent uniform dose $(E U D)$ of recurrent tumors. EUD for the original gross tumor volume (GTV), original planning tumor volume (PTV), recurrent gross tumor volume $(G T V)$, and recurrent planning tumor volume $(P T V)$ are shown for each of 18 patients (circle graphics, each color represents a different patient). The red line with the box graphic shows the overall mean EUD for that structure averaged for all 18 patients

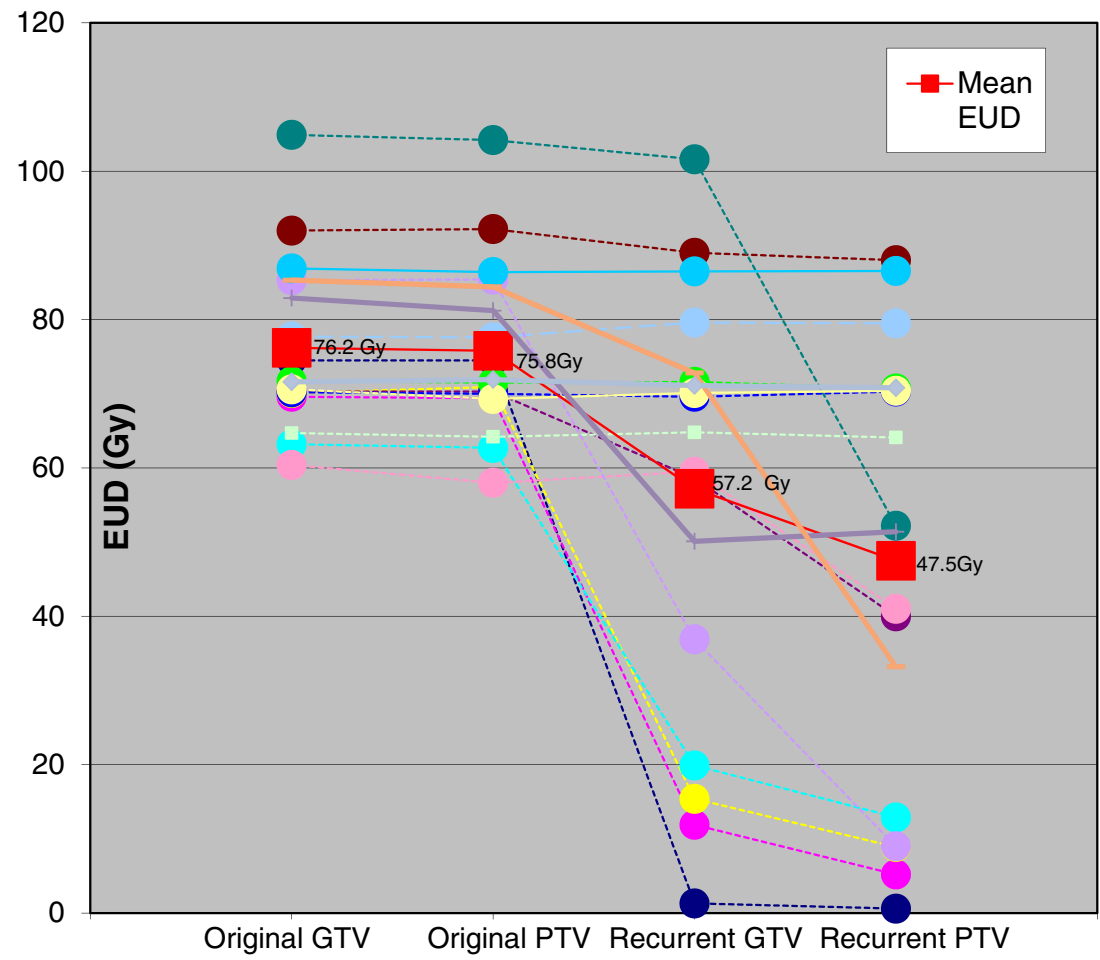


a

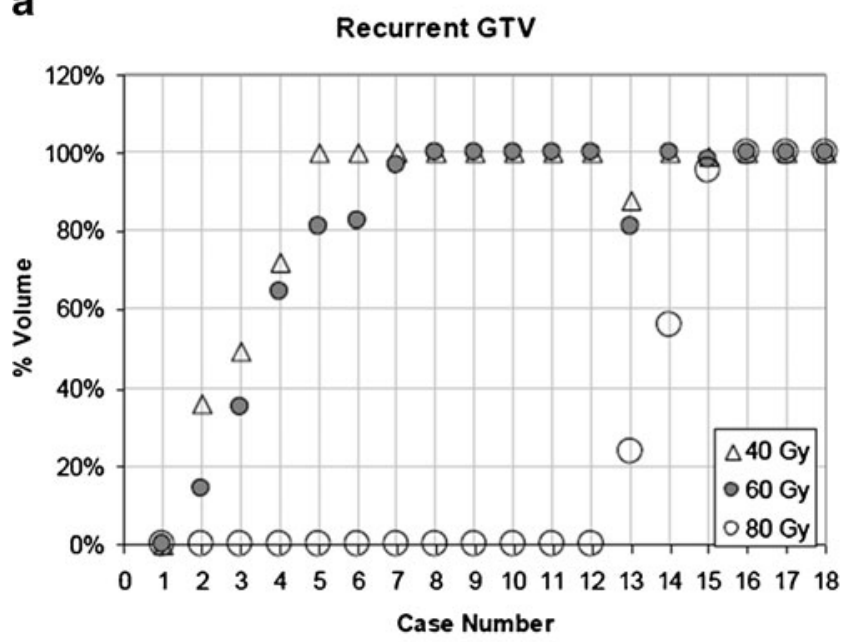

b

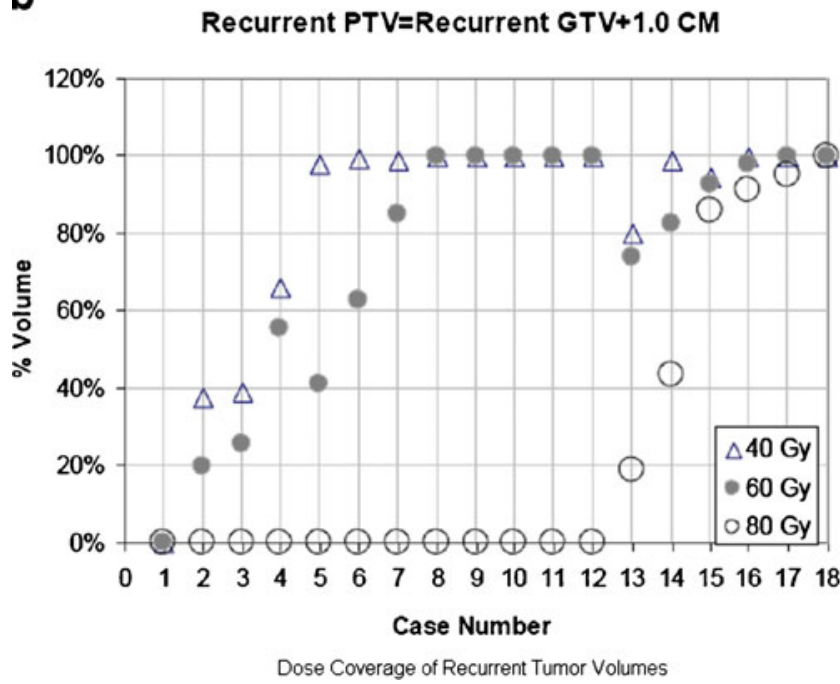

Fig. 2 a Dose to recurrent gross tumor volumes. This figure shows the percentage of each recurrent gross tumor volume (GTV) $(n=18$ patients) that receives 40,60 , or $80 \mathrm{~Gy}$. For example, the recurrent GTV for patient 13 (case \#13 on the horizontal axis) is $25 \%$ covered by the 80 -Gy isodose line (white circle icon), $80 \%$ covered by the 60 Gy isodose line (dark circle icon), and $90 \%$ covered by the $40-\mathrm{Gy}$ isodose line (white triangle icon). b Dose to recurrent planning target volumes. This figure shows the percentage of each recurrent planning target volume ( $P T V)(n=18$ patients) that receives 40,60 , or 80 Gy. For example, the recurrent PTV for patient 13 (case \#13 on the horizontal axis) is $20 \%$ covered by the $80-G y$ isodose line (white circle icon), $75 \%$ covered by the 60 -Gy isodose line (dark circle icon), and $80 \%$ covered by the 40-Gy isodose line (white triangle icon)

other dose calculation methodologies such as Monte Carlo dose calculation could have given different estimates of dose.

The second largest group failed peripherally (three patients) or marginally (four patients). For these patients, inadequate expansions for microscopic disease, tumor motion, or setup error could have resulted in under-dosing to areas at risk. At the time of this trial, modern 4D planning was not utilized during treatment planning or delivery. We assessed tumor motion with fluoroscopy at the time of simulation, but this is a suboptimal form of motion assessment. The outcome for these patients could well have been improved with the use of modern image-guided radiotherapy.

It is interesting to note that the recurrent tumor had significantly lower doses than the prescribed dose. This suggests that the origins of these recurrent tumors might not have received the prescription doses. Although image registration error cannot be excluded, it was most likely that part of original tumor moved outside of original PTV due to (1) tumor motion (as 4D CT was not used in this study), (2) larger than estimated setup error due to lack of image-guided technology at that time, or (3) dose computational errors. With the use of advanced technology such as modern motion assessment, image guidance during delivery, advances in computer technology/deformable image registration, and improvements in dose calculation algorithms, we anticipate this type of failure to decrease. It was also possible that the recurrent tumor may have not been included in the original PTV due to the limitations of using CT imaging for target delineation, as PET was not used for RT planning at that time. Pattern of failure analysis of RTOG 617 is awaited; all of the above are possible reasons. RTOG1106, a newly activated randomized trial, will use all these advanced technology in addition to PET guidance for target delineation.

The strength of this study is the unique insight gained from such intense dose escalation. Treating patients to doses in the 90-100-Gy range is uncommon, and outcomes from this treatment protocol can give us an interesting perspective on the dose-response relationship of non-small cell lung cancer to fractionated radiation therapy. In addition, because these patients were treated on a prospective protocol, their therapy was highly standardized and they underwent meticulous follow-up, which aids in this analysis.

The major limitation of this study is that we did not have CT data on all cases of recurrence and some planning CT scans were not recoverable. Another major limitation of this study is that lung anatomy can change over time as a result of disease and treatment. Lung consolidation, airway obstruction/pneumonia, and post-treatment fibrosis can cause anatomic distortion, which can shift the location of treated tissues and the original site of disease. These changes are dynamic over time and highly variable between patients. A certain degree of uncertainty about our estimates of treated tissues and doses are unavoidable because of these considerations. In addition, this trial was performed before concurrent chemotherapy had become the standard of care, and the patients in this trial were treated with either radiation alone or with sequential chemotherapy and radiation. Patients with early stage disease were also included in this trial, which differs from what would be done today.

In conclusion, despite very high doses of radiation, patients continue to experience high rates of locoregional failure. This highlights the importance of treatment technique and radiation technology to better control for these 
factors in future trials in order to ascertain the true benefit of dose escalation in this patient population.

Acknowledgments This work was supported in part by NIH grant P01 CA59827.

Conflict of interest The authors have no conflicts to disclose.

\section{References}

1. Machtay M, Bae K, Movsas B et al (2012) Higher biologically effective dose of radiotherapy is associated with improved outcomes for locally advanced non-small cell lung carcinoma treated with chemoradiation: an analysis of the radiation therapy oncology group. Int J Radiat Oncol Biol Phys 82(1):425-434

2. Rengan R, Rosenzweig KE, Venkatraman E et al (2004) Improved local control with higher doses of radiation in large-volume stage III non-small-cell lung cancer. Int J Radiat Oncol Biol Phys 60 (3):741-747

3. Wang L, Correa CR, Zhao L et al (2009) The effect of radiation dose and chemotherapy on overall survival in 237 patients with stage III non-small-cell lung cancer. Int J Radiat Oncol Biol Phys 73(5):1383-1390

4. Hayman JA, Martel MK, Ten Haken RK et al (2001) Dose escalation in non-small-cell lung cancer using three-dimensional conformal radiation therapy: update of a phase I trial. J Clin Oncol 19 (1):127-136

5. Narayan S, Henning GT, Ten Haken RK et al (2004) Results following treatment to doses of 92.4 or 102.9 Gy on a phase I dose escalation study for non-small cell lung cancer. Lung Cancer 44(1):79-88
6. Kong FM, Ten Haken RK, Schipper MJ et al (2005) High-dose radiation improved local tumor control and overall survival in patients with inoperable/unresectable non-small-cell lung cancer: long-term results of a radiation dose escalation study. Int $\mathrm{J}$ Radiat Oncol Biol Phys 63(2):324-333

7. Chen M, Hayman JA, Ten Haken RK et al (2006) Long-term results of high-dose conformal radiotherapy for patients with medically inoperable T1-3N0 non-small-cell lung cancer: is low incidence of regional failure due to incidental nodal irradiation? Int $\mathrm{J}$ Radiat Oncol Biol Phys 64(1):120-126

8. Kong FM, Hayman JA, Griffith KA et al (2006) Final toxicity results of a radiation-dose escalation study in patients with non-small-cell lung cancer (NSCLC): predictors for radiation pneumonitis and fibrosis. Int $\mathrm{J}$ Radiat Oncol Biol Phys 65 (4): $1075-1086$

9. Watkins JM, Wahlquist AE, Zauls AJ et al (2010) High-dose fractionated radiotherapy to $80 \mathrm{~Gy}$ for stage I-II medically inoperable non-small-cell lung cancer. J Med Imaging Radiat Oncol 54 (6):554-561

10. Chien CR, Chen SW, Hsieh CY et al (2001) Intra-thoracic failure pattern and survival status following 3D conformal radiotherapy for non-small cell lung cancer: a preliminary report. Jpn J Clin Oncol 31(2):55-60

11. Kumar P, Herndon J 2nd, Langer M et al (1996) Patterns of disease failure after trimodality therapy of nonsmall cell lung carcinoma pathologic stage IIIA (N2). Analysis of Cancer and Leukemia Group B Protocol 8935. Cancer 77(11):2393-2399

12. Tejedor M, Valerdi JJ, Lopez R et al (1995) Mitomycin, cisplatin, and vindesine followed by radiotherapy combined with cisplatin in stage III nonsmall cell lung cancer: long-term results. Int J Radiat Oncol Biol Phys 31(4):813-818

13. Timmerman R, Paulus R, Galvin J et al (2010) Stereotactic body radiation therapy for inoperable early stage lung cancer. JAMA 303(11): 1070 